\title{
Quantitative thermoelastic measurement on a helicopter glass fibre component underneath a surface anti-fretting coating.
}

\author{
A. Salerno ${ }^{1, a}$, A. Costa ${ }^{2}$ \\ ${ }^{1}$ Dipartimento di Energia, Politecnico di Milano, via Lambruschini 4, 20156 Milano, Italy \\ ${ }^{2}$ Vibrostop S.r.l., via Savona 125, 20144 Milano, Italy.
}

\begin{abstract}
Thermoelastic stress analysis (TSA) has been applied to measure the first stress invariant on a composite helicopter component under load. The component comprised inner mono-directional glass fiber layers with an outer central plate in glass fiber cloth, covered by an anti-fretting surface coating. In order to obtain quantitative results, a previous calibration of the thermoelastic constant obtained on a composite sample with a similar anti-fretting coating has been necessary.
\end{abstract}

\section{Introduction}

Thermoelastic stress analysis (TSA) can produce images of the stress distribution induced on the surface of a component under cyclic load, by acquiring infrared images of the surface temperature fluctuations of the component, produced by the thermoelastic effect. On composite materials, the achievement of quantitative TSA results is particularly challenging [1].

Fiber oriented composites are orthotropic materials in which the thermoelastic constant depends on the direction. The thermoelastic constant in a direction can be obtained as the composition of two thermoelastic constants, one along the direction of the fibers and the other in a transversal direction (Fig. 1), according to the formula [2]:

$$
\delta T=-\frac{T_{0}}{\rho C_{p}}\left(\alpha_{l} \delta \sigma_{l l}+\alpha_{t} \delta \sigma_{t t}\right)=-T_{0}\left(K_{l} \delta \sigma_{l l}+K_{t} \delta \sigma_{t t}\right)
$$

where $\delta T$ is the temperature variation, $T_{0}$ is the average temperature about which the thermoelastic temperature fluctuations are produced, $\rho$ is the mass density, $C_{p}$ the specific heat at constant pressure, $\alpha$ is the linear thermal elongation coefficient, $\delta \sigma$ is the stress variation and the subscripts $l$ and $t$ stand for longitudinal and transversal with respect to the direction of the fibers. In cases in which the composite is a cloth with fibers forming pick and warp, the thermoelastic constant does not depend on the direction.

\footnotetext{
a e-mail : antonio.salerno@polimi.it
} 


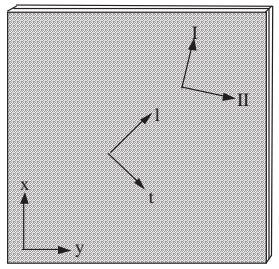

Fig. 1. Orthotropic material: transversal and longitudinal directions are indicated by letters " $\mathrm{l}$ " and " $\mathrm{t}$ ".

Another problem in quantitative TSA on composites is the presence of a thin layer of superficial resin free of fibers, whose thickness is far from being uniform (Fig. 2). Such a layer does not contribute to the structural resistance of the component, but it modifies the amplitude and the phase of the thermal waves generated in the component by the thermoelastic effect, thus creating a dependence of the thermoelastic constant on the frequency of the modulated load [3].

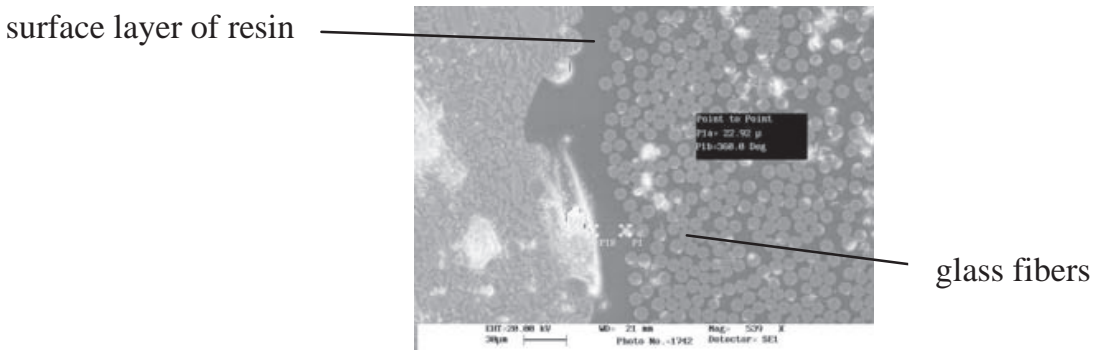

Fig. 2. Microscope image of a section of a glass fiber composite

\section{Description of the component and the loads applied}

The helicopter component to be inspected comprised in the central region fourteen inner monodirectional glass fiber layers, on the surface of which there was a superficial plate in glass fiber cloth, covered by an anti-fretting surface coating. In Fig. $3 \mathrm{a}$ is visible the component mounted on the test bench, while in Fig. $3 \mathrm{~b}$ is shown the central part of the component with the four bolts fixing it to the test bench. The reinforcing plate is not directly visible because covered by the anti-fretting coating.

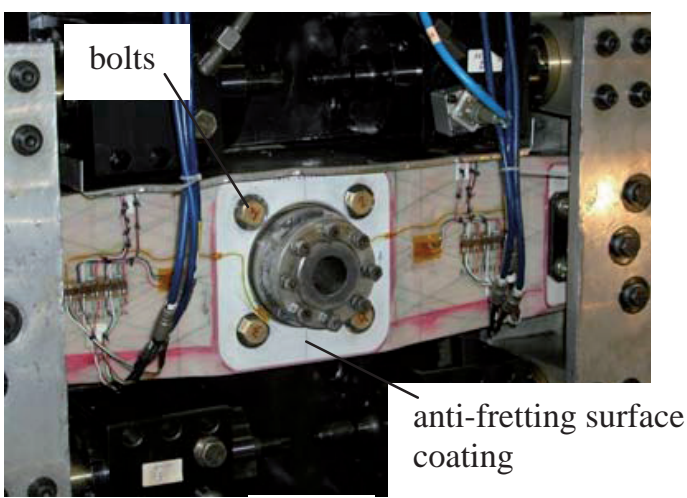

a)

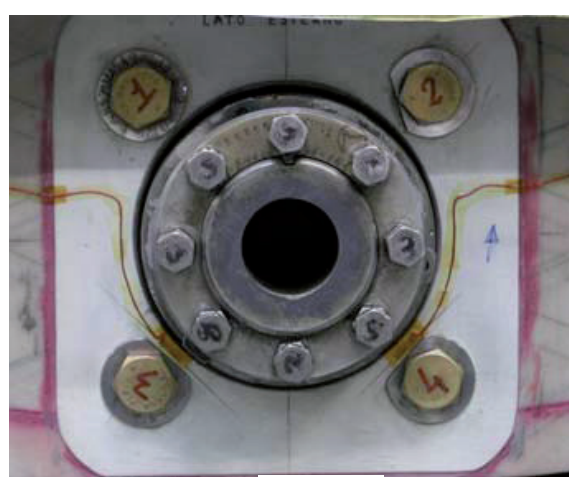

b)

Fig. 3. Component mounted on its test bench 
The superficial plate made in glass fiber cloth behaves as an isotropic material from a thermoelastic point of view.

The anti-fretting coating, made of an isotropic polymeric material, had a thickness of $0.25 \mathrm{~mm}$, thus being capable of influencing the thermoelastic measurement.

Two strain gauges were placed on the surface of the anti-fretting coating but they were not able to give any significant result except a reference signal to perform the TSA. The reason for this will be discussed in paragraph 7 .

Three different modulated loads (Tab.1), reproducing the operational loads, were alternatively applied to the component as shown in Fig. 4. For load B the control was on the displacement.

Table 1. Loads applied to the component.

\begin{tabular}{|l|c|c|}
\hline \multicolumn{1}{|c|}{ Load } & Static & Dynamic \\
\hline A: Centrifugal & $33800 \mathrm{~N}$ & $27650 \mathrm{~N}$ \\
\hline B: Bending out of plane & $8 \mathrm{~mm}$ & $8 \mathrm{~mm}$ \\
\hline C: Bending in plane & $-20000 \mathrm{~N}$ & $7000 \mathrm{~N}$ \\
\hline
\end{tabular}

The loads were modulated at a frequency of $1 \mathrm{~Hz}$.

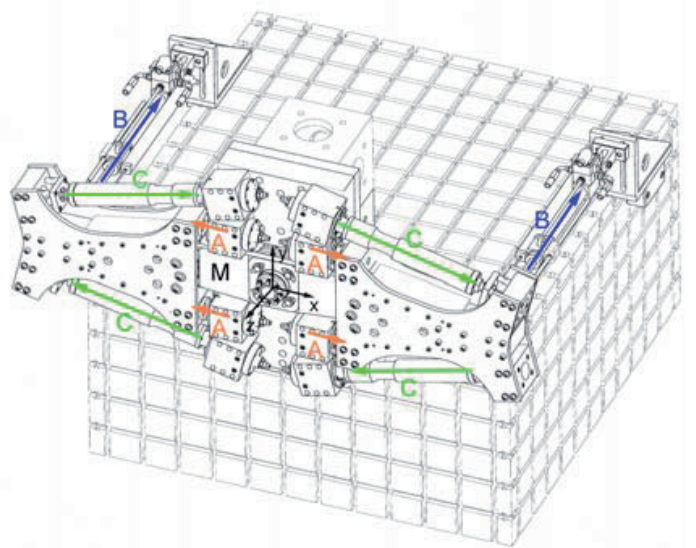

Fig. 4. Loads applied to the component

The TSA measurement was aimed at measuring the stress distribution on the reinforcing plate underneath the anti-fretting coating.

\section{Calibration of the thermoelastic constant.}

The anti-fretting coating on the component surface is a non structural element which produces an attenuation and a phase shift of the thermoelastic signal, thus introducing an error in a quantitative measurement of the local stress.

A calibration procedure, performed on a mono-directional glass fibers sample coated with the same anti-fretting material, was conducted in order to relate the stress induced in the glass fiber sample with the temperature on the surface of the anti-fretting coating. This solution is based on two reasons:

a) the stress induced in the anti-fretting coating cannot be easily evaluated because, though the strain can be measured, the Young modulus is not precisely known 
b) only the evaluation of the stress in the reinforcing plate in glass fiber cloth and not that in the anti-fretting coating is interesting, from a structural point of view.

Fig. 5 shows the glass fiber sample with the $0.25 \mathrm{~mm}$ thick anti-fretting coating on its surface in the visible (a) and in the infrared (b). On the surface are indicated the points on the anti-fretting coating used for the calibration and the reference point $\mathrm{R}$ outside the anti-fretting coating.

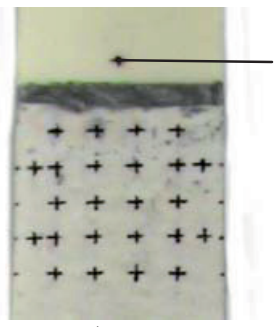

a)

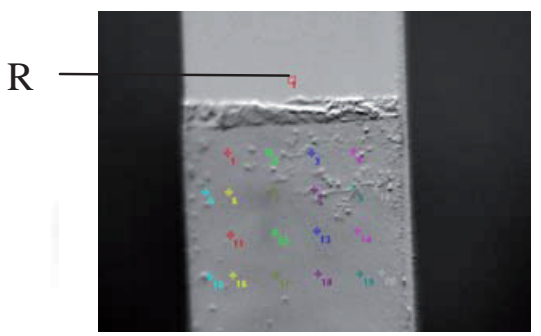

b)

Fig. 5. Glass fiber sample with the anti-fretting coating used for the calibration of the thermoelastic constant (a: image taken in the visible, b: image taken in the infrared)

The sample was loaded with a longitudinal mono-axial load of $1650 \pm 1350 \mathrm{~N}$. Though the antifretting coating was $7.5 \%$ of the sample thickness, his low Young modulus (evaluated to be at least six time less than that of the glass fiber sample) produced a negligible influence on the stress in the glass fiber material underneath (less than 3\%). Fig. 6 shows the experimental temperature values obtained at different frequencies on the anti-fretting coating as an average of the measurement on several points on the coating and outside the coating on point $\mathrm{R}$ of Fig. 5. The attenuation produced by the coating is evident.

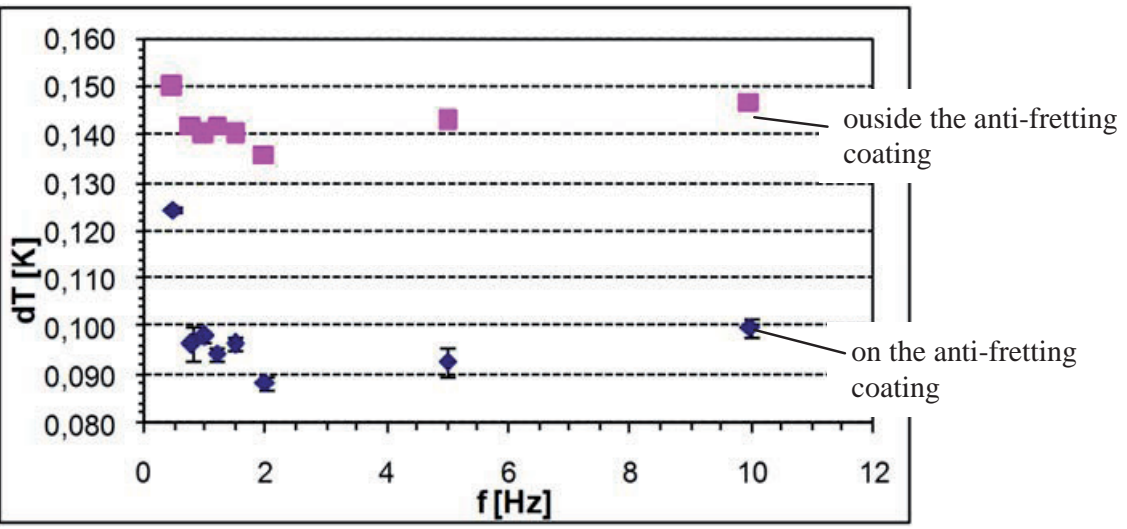

Fig. 6. Experimental temperature values obtained on the calibration sample at different frequencies

The peak to peak stress induced in the sample was evaluated to be $\delta \sigma=34.4 \mathrm{MPa}$ on the base of the load applied and the cross section of the sample. This value, together with the measured temperature variation $d T$ and average temperature $T_{0}$ allowed to reckon the thermoelastic constant for this particular configuration using the relation:

$$
K=d T /\left(T_{0} \delta \sigma\right)
$$


The value of the thermoelastic constant vs. frequency is represented in Fig. 7, where it is clearly visible a dependence of the thermoelastic constant on the frequency, especially at $0,5 \mathrm{~Hz}$.

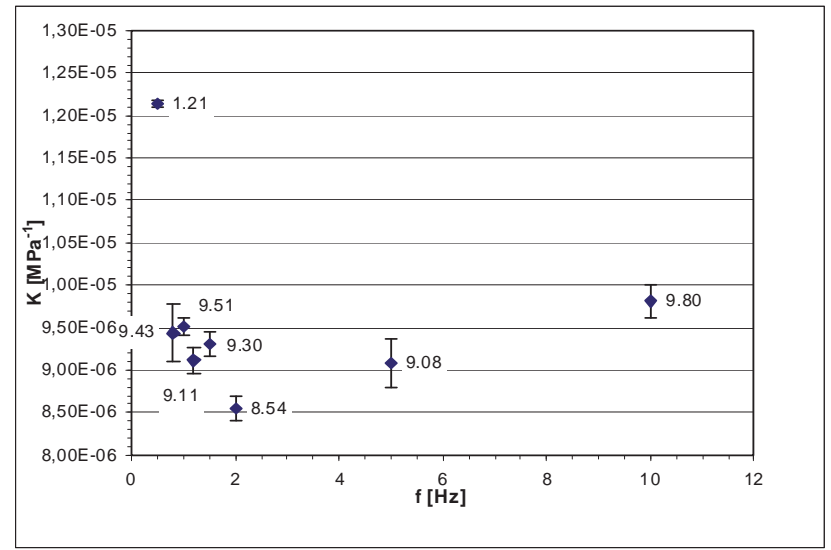

Fig. 7. Thermoelastic constant vs. frequency

\section{Evaluation of the influence on the thermoelastic signal of the mono- directional layers underneath the reinforcing plate in fiber glass cloth.}

The inner mono-directional layers can be subject to a stress value, which is different from that of the superficial reinforcing plate in fiber glass cloth. Thermal waves generated by the TSA effect present a damping coefficient which depends on their frequency. If the TSA test is performed at a not sufficiently high frequency, the thermal waves generated in the inner layers can reach the surface passing through the reinforcing plate, thus influencing the measurement. In this case adiabatic conditions are not reached and the thermoelastic signal on the surface depends on the frequency of the load applied. In order to investigate this possible source of error, a simple mono-dimensional model was made simulating the seven mono-directional layers (till the central line of symmetry) and the reinforcing plate on their surface. model.

Fig. 8 shows the surface temperature variation $d T$ and the phase shift obtained from this
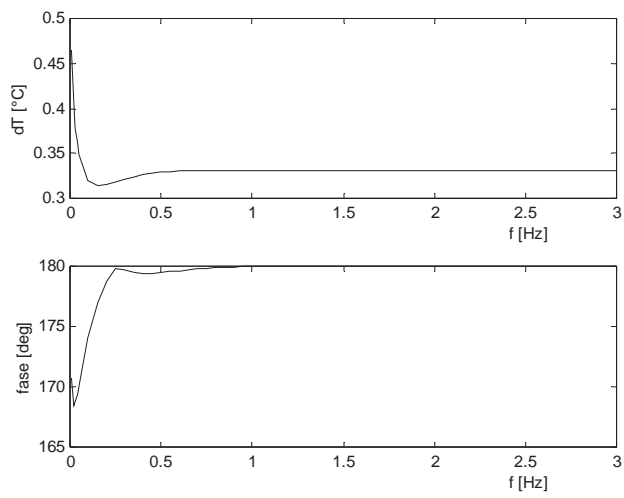

Fig. 8. Surface temperature variation and phase shift obtained from the model.

The results obtained from the model show that adiabatic conditions are reached for frequencies above $0,5 \mathrm{~Hz}$. Tests realized at $1 \mathrm{~Hz}$ are therefore not affected by this source of error. 


\section{Results}

The results obtained for the three different loads are shown and discussed in the following three paragraphs. These results were then compared with the results obtained by a simple finite element model.

\subsection{Centrifugal load}

The stress in the central part of the component under centrifugal load is limited by the presence of the four bolts attaching the component to the test bench (Fig. 9).

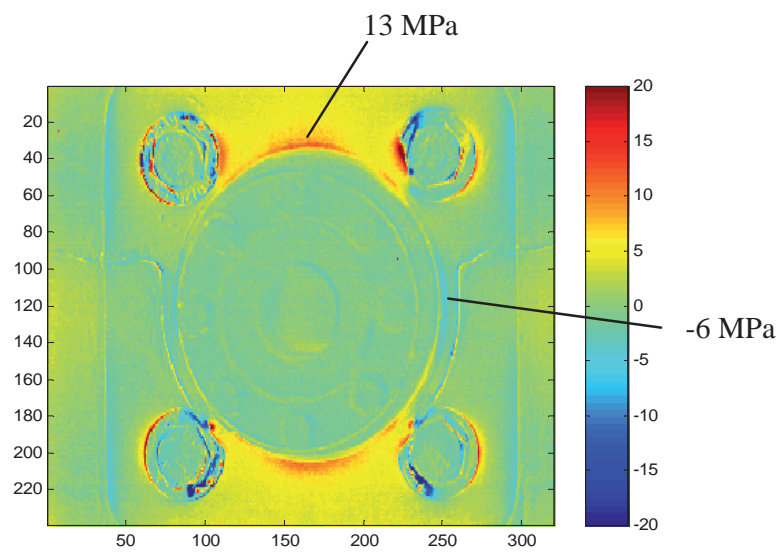

Fig. 9. TSA obtained for the centrifugal load applied at $1 \mathrm{~Hz}$

A cut in the washer allowed to visualise a stress concentration of $30 \mathrm{MPa}$ close to the bolt (Fig. $10)$.

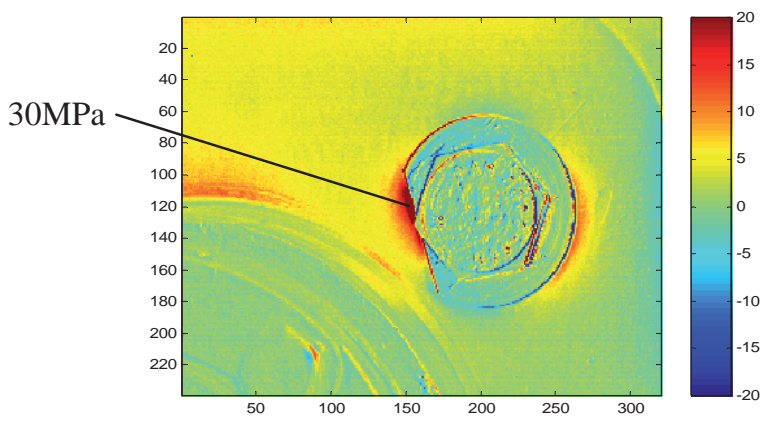

Fig. 10. Detail close to the bolt

\subsection{Bending out of plane}

In the TSA image obtained in the bending out of plane, a clear difference of the stress concentration in the upper part and in the lower part of the image shows the presence of an unexpected downward component in the load applied (Fig. 11). 


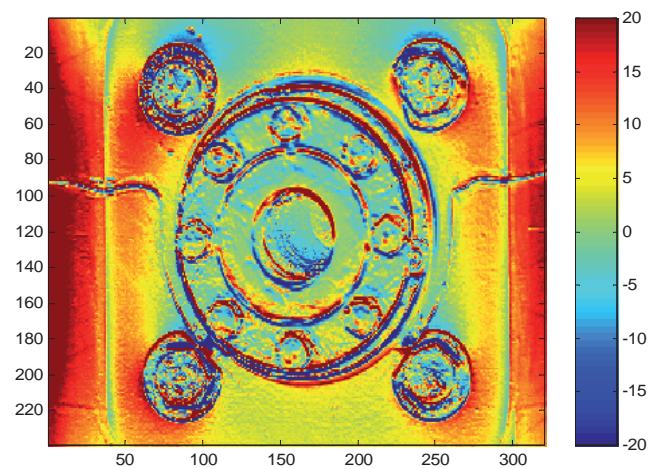

Fig. 11. TSA obtained for the load of bending out of plane

\subsection{Bending in plane}

Under the bending in plane the stress is concentrated around the bolts. The two strain gauges are positioned in a region of sign change for the stress.

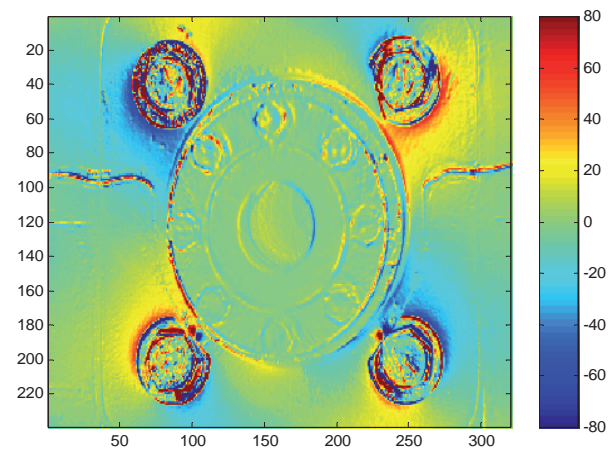

Fig. 12. TSA obtained for the load of bending in plane

\section{FEM model}

A simple FEM model was used to compare the results obtained experimentally. Fig. 13 shows the results obtained by the FEM model applying the centrifugal load.

The model had been originally realised to study the edges of the component. For this reason the mesh in the central part corresponding to the reinforcing plate was very simple and the bolts were not modelled. Their effect was simulated with a nodal constraint in the corresponding positions. Furthermore the roughness of the mesh and its not perfect symmetry, with respect to a longitudinal centre line, were also responsible for the non perfect symmetry of the results. 

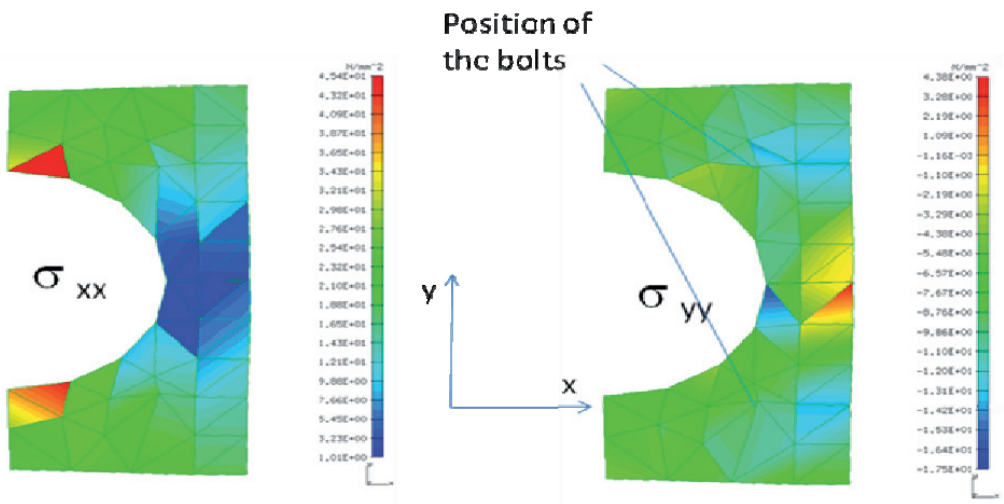

Fig. 13. Results for the centrifugal load

\section{Discussion of the results}

The absence of the bolts in the FE model did not allow a comparison of the results for the load of bending in plane, in which the stress is mainly concentrated around the bolts. Also in the case of the bending out of plane, the presence of an asymmetry in the real load allowed only for a partial comparison of the results. For the centrifugal load there was a good agreement of the results in regions far from the bolts. For example on the vertical axis, passing from the centre of the component, the difference between the FEM and the TSA results was comprised between 1.9 and 5.2 MPa.

The presence of two strain gauges visible in Fig. 3 did not give useful results. One problem was that their size, approximately $4 \mathrm{~mm}$, did not allow to precisely read the value of the stress in regions with high stress gradients. Furthermore, in the bending in plane and in the centrifugal load their position corresponded to a region of sign transition for the stress. In the bending out of plane the deformation of the strain gauges, placed on the surface of the anti-fretting coating, did not correspond to the deformation of the reinforcing plate underneath.

\section{Conclusion}

In the study of a glass fibre helicopter component the TSA allowed for the quantitative evaluation of the stress distribution induced by three different loads in a central glass fibre reinforcing plate covered by an anti-fretting coating.

The comparison of the TSA results with numerical results obtained by a simple FE model showed a good agreement, in cases in which this comparison was possible.

The validation of the TSA results using the two strain gauges disposed on the anti-fretting coating was not possible due to their positioning.

\section{References}

1. A. K. Wong, J. Phys. Chem. Solids 52, 483 (1991)

2. P. Stanley, W. K. Chan, J. Strain Anal. 23, 137 (1988)

3. A. Salerno, A. Costa, G. Fantoni, Rev. Sci. Instr. 80, 034904 (2009) 